Geometry \& Topology

Volume 9 (2005) 1221-1252

Published: 24 July 2005

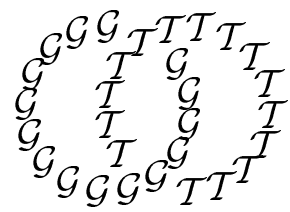

\title{
The nonuniqueness of Chekanov polynomials of Legendrian knots
}

\author{
PAul Melvin \\ Sumana Shrestha \\ Department of Mathematics, Bryn Mawr College \\ Bryn Mawr, PA 19010, USA
}

Email: pmelvin@brynmawr.edu

\begin{abstract}
Examples are given of prime Legendrian knots in the standard contact 3-space that have arbitrarily many distinct Chekanov polynomials, refuting a conjecture of Lenny Ng. These are constructed using a new "Legendrian tangle replacement" technique. This technique is then used to show that the phenomenon of multiple Chekanov polynomials is in fact quite common. Finally, building on unpublished work of Yufa and Branson, a tabulation is given of Legendrian fronts, along with their Chekanov polynomials, representing maximal ThurstonBennequin Legendrian knots for each knot type of nine or fewer crossings. These knots are paired so that the front for the mirror of any knot is obtained in a standard way by rotating the front for the knot.
\end{abstract}

\section{AMS Classification numbers Primary: 57R17}

Secondary: 57M25, 53D12

Keywords: Legendrian knots, contact homology, Chekanov polynomials

Proposed: Yasha Eliashberg

Seconded: Robion Kirby, Joan Birman
Received: 10 November 2004

Revised: 3 December 2004 


\section{Introduction}

A smooth knot $K$ in $\mathbb{R}^{3}$ is Legendrian if it is everywhere tangent to the twoplane distribution $\operatorname{ker}(\alpha)$ of the standard contact one-form $\alpha=d z-y d x$. It is tacitly assumed that the projection of $K$ onto the $x z$-plane, its front, is generic, that is all self-intersections of the front are transverse double points. Two Legendrian knots are Legendrian isotopic if they are smoothly isotopic through (not necessarily generic) Legendrian knots. Much of the recent work in Legendrian knot theory has been motivated by the problem of classifying Legendrian knots up to Legendrian isotopy within a fixed knot type [12].

The two "classical" Legendrian isotopy invariants of a Legendrian knot $K$ are its Thurston-Bennequin number $t b(K)$ and its (absolute) rotation number $r(K)$. They can be computed by the formulas

$$
t b(K)=w-c / 2 \quad r(K)=|u-d| / 2
$$

where $w$ is the writhe of the front of $K$ (the difference of the number of positive and negative crossings) and $c$ is the number of cusps in the front, of which $u$ are traversed upward and $d$ are traversed downward with respect to any chosen orientation on $K$. These two invariants serve to classify the Legendrian knots in some knot types, including the unknot [7, the figure eight and all torus knots [9. Such knot types are called Legendrian simple.

There are many knot types that are not Legendrian simple. The first examples, discovered independently by Chekanov [3] and Eliashberg-Hofer [6], were the positive twist knots (other than the trefoil and figure eight). The inequivalence of suitable Legendrian representatives of these knots (with identical classical invariants) was detected using contact homology [16, 8], a version of Floer homology adapted to contact manifolds. More recently, convex surface techniques have been used to detect other examples among the iterated torus knots [1].

Chekanov's approach to contact homology is purely combinatorial, and yields an easily computable family of integer Laurent polynomial invariants $P_{\varepsilon}(t)$ indexed by the augmentations $\varepsilon$ of (the differential graded algebra of) $K$; see Section 2 for the definitions. These will be called the Chekanov polynomials of $K$, and the number $\operatorname{ch}(K)$ of distinct such polynomials will be called the Chekanov number of $K$.

It has been observed by Chekanov [4, Section 2.2], Ng [20, Section 3.16] and [21, and others that computational evidence (at the time) supported the following conjecture. 
Conjecture (Uniqueness of Chekanov polynomials) The Chekanov number $\operatorname{ch}(K)$ of any Legendrian knot $K$ is at most 1.

In this paper it is shown that this conjecture fails. The first counterexample we discovered, representing the mirror of the knot type $8_{21}$, has Chekanov number 2. Knots with arbitrarily large Chekanov numbers can then be constructed using connected sums. With a little more work, prime knots of this kind can be found.

Theorem 1.1 For every integer $k \geq 0$, there is a prime Legendrian knot with Chekanov number $k$.

The proof is given in Section 3, and a conceptual explanation of why these examples arise is given in Section 4 using a Legendrian tangle replacement method. It should be noted that these knots also provide counterexamples to the more speculative conjecture (cf [4, Section 2.2]) that the ranks $P(1)$ are equal for all Chekanov polynomials $P$ of a given Legendrian knot $K$; for the knots in question, these ranks are in fact all distinct. In contrast, it is an easy exercise to show that the Euler characteristic $P(-1)=t b(K)$ for any Chekanov polynomial $P$ of $K$.

Our computations throughout are simplified by a beautiful recent result of Josh Sabloff [25]:

Duality Theorem Any Chekanov polynomial of a Legendrian knot can be written in the form

$$
P(t)=t+p(t)+p\left(t^{-1}\right)
$$

for some (honest) polynomial $p(t)$ with positive integer coefficients.

Such a polynomial $p$ will be called a reduced Chekanov polynomial of the knot. Using this result and elementary observations about connected sums of Legendrian knots, we give (in Section 3i) a characterization of reduced Chekanov polynomials.

Theorem 1.2 Every polynomial with positive integer coefficients arises as the reduced Chekanov polynomial of some Legendrian knot.

It is an interesting problem to determine when $c h(K)$ is nonzero. Of course this can be solved algorithmically, since the augmentations of $K$ are the solutions of a system of polynomial equations over $\mathbb{Z}_{2}$ that can be read off from the front of 
$K$ (see Section 2). Unfortunately, the known algorithms for solving this system are all of exponential complexity. It would be useful to find more computable criteria.

Fuchs and Ishkhanov [15], and independently Sabloff [24], have found a useful geometric criterion that is equivalent to the existence of an augmentation, namely the existence of a ruling in the sense of Chekanov and Pushkar [4] [5]. The obvious algorithms for finding a ruling, however, are still of exponential complexity.

Another necessary (but far from sufficient) condition for the existence of an augmentation is that $K$ be nondestabilizable, ie not Legendrian isotopic to a front with a kink $\infty$. An important class of such knots are the ones of maximal Thurston-Bennequin number in their knot types, which we simply call maximal knots. (Note that there exist nondestabilizable knots that are not maximal 11.) All the knots tabulated in Section 5 are maximal. Less that half of them, however, have augmentations. Moreover, there is no known algorithm for deciding in the first place if a knot is maximal (or nondestabilizable).

A less obvious (but readily computable) necessary condition is the vanishing of the rotation number $r(K)$. This was surmised from our calculations in Section [5] and confirmed by Sabloff as an easy consequence of the existence of a ruling [24].

Proposition 1.3 If $r(K) \neq 0$, then $\operatorname{ch}(K)=0$.

Most of the knots with rotation number zero tabulated below have augmentations, although presumably this becomes less prevalent with increasing crossing number. Among these is the knot of type $7_{4}$, of Chekanov number 1 . In contrast, $\mathrm{Ng}$ [20. Section 4.2] has given examples of knots in this knot type, with the same classical invariants, which do not have augmentations. Thus the Chekanov number can be used to distinguish knots of the same type with the same classical invariants.

Note that in general, $t b(K)$ and $r(K)$ are of opposite parity; this can be proved in variety of ways, for example by induction on the number of crossings in the front of $K$. It follows from the Proposition that only knots with odd ThurstonBennequin invariant can have augmentations.

It remains an open problem to find complete criteria, computable in polynomial time, for the existence of an augmentation.

Acknowledgments We are grateful to Josh Sabloff for introducing us to this subject through his beautiful lectures in the TriCo Contact Seminar, and for 
providing us with an early version of his book on Legendrian knots. We would also like to thank Lisa Traynor for stimulating discussions and graphics advice. The first author is partially supported by National Science Foundation grant FRG-0244460.

\section{Chekanov polynomials}

Let $K$ be a Legendrian knot with rotation number zero. For computational convenience we assume following $\mathrm{Ng}$ [19] that the front of $K$ is simple, ie all the right cusps have the same $x$-coordinate. This can be arranged by a Legendrian isotopy of $K$. Any smooth path in the front joining a left and right cusp will be called a spanning arc of $K$ (so if there are $c$ cusps, then the front consists of $c$ spanning arcs). Any connected component of the complement of the front in the $x z$-plane will be called a region in the front, and a disk formed from the closure of a union of regions will be called a disk with corners. The corners refer to the nonsmooth points in the boundary that occur at right cusps or crossings.

Now recall the definition of Chekanov's differential graded algebra $(\mathcal{A}, \partial)$ for $K$. The underlying algebra $\mathcal{A}$ is the free noncommutative algebra over $\mathbb{Z}_{2}$ generated by the crossings $c_{1}, \ldots, c_{n}$ and right cusps $c_{n+1}, \ldots, c_{n+r}$ in the front. Thus the elements of $\mathcal{A}$ are finite sums of words in the $c_{i}$, where the empty word, denoted by 1 , is the identity. The full set $\left\{c_{1}, \ldots, c_{n+r}\right\}$ of generators will be denoted by $\mathcal{C}$.

The grading on $\mathcal{A}$ is defined by assigning an integer degree $|c|$ to each $c \in \mathcal{C}$, and then extending to higher order terms by the rule $|a b|=|a|+|b|$. To define $|c|$, choose a Maslov potential $\mu$ on the front of $K$. By definition, $\mu$ assigns a real number to each spanning arc in the front in such a way that the upper arc at any cusp is assigned one more than the lower arc; such an assignment can be made consistently since $r(K)=0$. Now set $|c|=1$ if $c$ is a cusp, and

$$
|c|=\mu(\alpha)-\mu(\beta)
$$

if $c$ is a crossing, where $\alpha$ is the upper (smaller slope) arc at the crossing and $\beta$ is the lower arc. We will denote the subset of $\mathcal{C}$ of generators of degree $k$ by $\mathcal{C}_{k}$, and its cardinality by $n_{k}$.

Finally the differentials $\partial c$ for $c \in \mathcal{C}$ are defined using suitable embedded disks, and then extended by the Leibnitz rule and linearity, setting $\partial 1=0$, to all of $\mathcal{A}$. (If the front is not simple, one must consider immersed disks.) In particular, a disk $D$ with corners is called an admissible disk for $c$ if its right-most corner 
is at $c$, its left-most point is at a left cusp, and the remaining corners, called the negative corners of $D$ for reasons explained in [23, are convex (meaning that they occur at crossings that lie on the boundary of exactly one of the regions in $D$ ). There is an associated monomial $\partial D \in \mathcal{A}$ obtained by reading off the labels at the negative corners of $D$, proceeding from $c$ counterclockwise around the boundary. Now define

$$
\partial c= \begin{cases}\sum \partial D & \text { if } c \text { is a crossing } \\ 1+\sum \partial D & \text { if } c \text { is a right cusp }\end{cases}
$$

where the sums are over all admissible disks $D$ for $c$. It is not difficult to show that $\partial$ lowers degree by 1 , and that $\partial^{2}=0$. The graded homology $H_{*}(\mathcal{A}, \partial)$, which coincides with the contact homology of the standard contact $\mathbb{R}^{3}$ relative to $K$ [13], is invariant under Legendrian isotopy of $K$.

The algebra $(\mathcal{A}, \partial)$ is infinite dimensional (over $\left.\mathbb{Z}_{2}\right)$ and generally cumbersome to deal with as a whole, but useful information can be extracted from its finite dimensional quotients. In particular, consider projections $\pi_{*}: \mathcal{A} \rightarrow C$ where $C$ is the finite dimensional space spanned by $\mathcal{C}$. Under suitable conditions the induced endomorphism $\partial_{*}=\pi_{*} \partial$ of $C$ is a differential (that is $\partial_{*}^{2}=0$ ) and the associated Poincaré polynomial $P_{*}(t)=\sum_{k} \operatorname{dim}\left(H_{k}\left(C, \partial_{*}\right)\right) t^{k}$ is a Legendrian isotopy invariant. The Chekanov polynomials $P_{\varepsilon}$ arise in this way from projections $\pi_{\varepsilon}: \mathcal{A} \rightarrow C$ associated with augmentations $\varepsilon$ of $(\mathcal{A}, \partial)$. Here are the details.

Definition 2.1 An augmentation of $(\mathcal{A}, \partial)$ (also called an augmentation of $K)$ is an algebra map

$$
\varepsilon: \mathcal{A} \rightarrow \mathbb{Z}_{2}
$$

that vanishes on elements of nonzero degree and satisfies $\varepsilon \partial=0$. The generators $c \in \mathcal{C}$ with $\varepsilon(c)=1$, which are all crossings of degree zero, will be called the augmented crossings of $\varepsilon$.

The associated projection $\pi_{\varepsilon}$ is defined on monomials $m=c_{i_{1}} \ldots c_{i_{k}}$ (which form a basis for $\mathcal{A}$ over $\mathbb{Z}_{2}$ ) by extracting the linear term in

$$
\left(c_{i_{1}}+\varepsilon\left(c_{i_{1}}\right)\right) \ldots\left(c_{i_{k}}+\varepsilon\left(c_{i_{k}}\right)\right)
$$

where $\pi_{\varepsilon}(1)=0$ by convention. Thus

$$
\pi_{\varepsilon}(m)=c_{i_{1}}+\cdots+c_{i_{k}}
$$

if all the factors $c_{i_{j}}$ are augmented. Such a monomial will be called pure. If all but one of the factors, say $c$, are augmented, then $\pi_{\varepsilon}(m)=c$. In this case we say that $m$ is full. In all other cases $\pi_{\varepsilon}(m)=0$. 
The projection $\pi_{\varepsilon}$ induces a graded differential $\partial_{\varepsilon}=\pi_{\varepsilon} \partial$ on $C$, or more precisely a chain complex

$$
\cdots \longrightarrow C_{k+1} \stackrel{\partial_{k+1}}{\longrightarrow} C_{k} \stackrel{\partial_{k}}{\longrightarrow} C_{k-1} \longrightarrow \cdots
$$

where $C_{k}$ is the vector space spanned by the set $\mathcal{C}_{k}$ of generators of degree $k$, and $\partial_{k}=\partial_{\varepsilon} \mid C_{k}$. Explicitly, the differential $\partial_{\varepsilon} b$ for any $b \in \mathcal{C}$ is the sum of all the factors in all the pure monomials in $\partial b$ added to the unaugmented factors in the full monomials in $\partial b$.

The Chekanov polynomial $P_{\varepsilon}$ is the Poincaré polynomial of this complex,

$$
P_{\varepsilon}(t)=\sum \operatorname{dim}\left(H_{k}\right) t^{k}
$$

where $H_{k}=\operatorname{ker} \partial_{k} / \operatorname{im} \partial_{k+1}$.

Remark 2.2 An augmentation $\varepsilon$ is uniquely determined by its set of augmented crossings, which by abuse of notation will also be denoted by $\varepsilon$. The condition $\varepsilon \partial=0$ simply asserts that (the set) $\varepsilon$ is the support of a solution to the system $\{\partial c=0: c \in \mathcal{C}\}$ of (commutative) polynomial equations over $\mathbb{Z}_{2}$. Thus the set $\operatorname{Aug}(K)$ of all augmentations can be viewed as the set of all subsets of $\mathcal{C}_{0}$ which are supports of solutions to this system.

This condition can be interpreted in simple geometric terms as follows: Start with an arbitrary subset $\varepsilon$ of $\mathcal{C}_{0}$. In analogy with the terminology above, we say that an admissible disk for a generator $c \in \mathcal{C}$ is a pure disk (with respect to $\varepsilon$ ) if all of its negative corners are in $\varepsilon$, and full if all but one are in $\varepsilon$. Let $|c\rangle \in \mathbb{Z}_{2}$ denote the mod 2 reduction of either the number of pure disks for $c$, or one more than that number, according to whether $c$ is a crossing or a right cusp. Then, noting that $|c\rangle=0$ if $|c| \neq 1$, we have

$$
\varepsilon \in \operatorname{Aug}(K) \Longleftrightarrow|c\rangle=0 \text { for all } c \in \mathcal{C}_{1} \text {. }
$$

In the same vein, the differential $\partial_{\varepsilon}$ for an augmentation $\varepsilon$ can be defined by

$$
\partial_{\varepsilon} c=\sum_{b}\langle b \mid c\rangle b
$$

where $\langle b \mid c\rangle$ is the number of admissible disks for $c$ which have a corner at $b$ and for which all remaining corners are augmented. (These disks are all pure if $b$ is augmented, and all full otherwise.)

Remark 2.3 The coefficient of $t^{k}$ in $P_{\varepsilon}(t)$ can be expressed as

$$
n_{k}-r_{k}-r_{k+1}
$$


where $n_{k}=\operatorname{dim} C_{k}=\left|\mathcal{C}_{k}\right|$ and $r_{k}=\operatorname{rk} \partial_{k}$. It follows that $P_{\varepsilon}$ is determined by the ranks $r_{k}$ for all $k>0$. Indeed these give the coefficients for all $k>0$, and thence for $k<0$ by the Duality Theorem (see Section 11). The constant coefficient is then computed using the identity $P_{\varepsilon}(-1)=\sum_{k}(-1)^{k} n_{k}$. Note that this last sum also computes $t b(K)$, which is odd since $r(K)=0$ is even.

By the same argument $P_{\varepsilon}$ is determined by the ranks $r_{k}$ for all $k<0$. Thus if $|c| \geq-1$ for all $c \in \mathcal{C}$, as for the examples in the next section, then the reduced polynomial $p_{\varepsilon}$ is linear and is determined by the single rank $r_{0}$ :

$$
p_{e}(t)=a t+b
$$

where $a=n_{-1}-r_{0}$ and $b=a+(t b(K)+1) / 2$. In particular, if there are no crossings of negative degree then $p_{\varepsilon}$ is constant.

\section{$3 \quad$ Examples}

First a familiar computation. Let $K_{1}$ be the Legendrian trefoil whose front is shown in Figure 1.

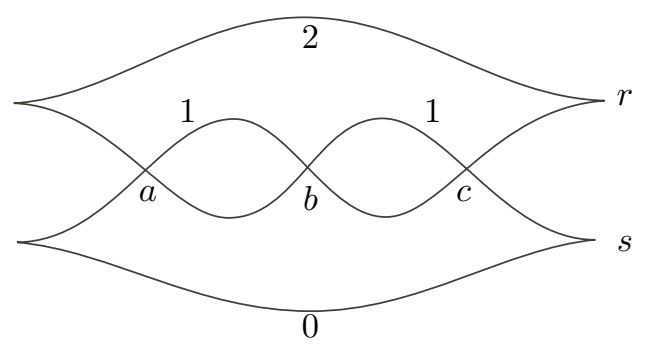

Figure 1: Front for the knot $K_{1}$ representing the right-handed trefoil

The graded generating sets are $\mathcal{C}_{1}=\{r, s\}$ (the right cusps) and $\mathcal{C}_{0}=\{a, b, c\}$ (computed using the Maslov potential indicated by the numbers above each arc in the diagram). The differentials of $a, b$ and $c$ vanish since there are no admissible disks for these crossings, while

$$
\partial r=1+[a b c] \text { and } \partial s=1+[c b a]
$$

where by definition $[x y z]=x+x y z+z$.

The augmentations of $K_{1}$ correspond to solutions to the system $\partial r=\partial s=$ 0 , or equivalently to the single equation $[a b c]=1$ (since $[a b c]=[c b a]$ after abelianizing). By inspection

$$
\operatorname{Aug}\left(K_{1}\right)=\{a b c, a b, a, b c, c\}
$$


where for notational economy we write $a b c, a b, \ldots$ for the sets $\{a, b, c\},\{a, b\}, \ldots$ (These five subsets will be referred to below as the admissible subsets of the ordered set or "triple" $a b c$.) By direct computation, or an appeal to the duality theorem as in Remark 2.3 above, all of these augmentations yield the same Chekanov polynomial $t+2$, or equivalently the same reduced polynomial 1 . In particular, $K_{1}$ has Chekanov number 1.

Next consider the Legendrian knot $K_{2}$ given by the front in Figure 2, which is topologically the mirror image of the knot $8_{21}$ (also denoted $\overline{8}_{21}$ ).

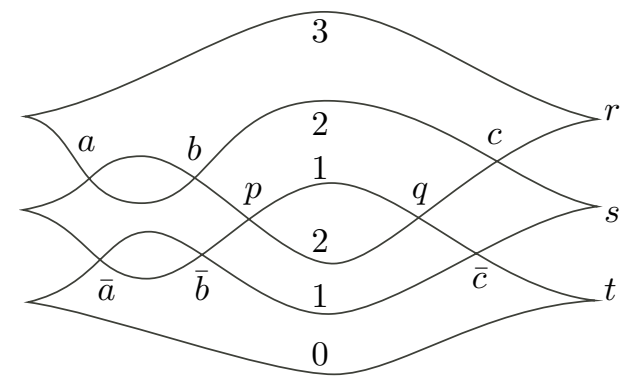

Figure 2: Front for the knot $K_{2}$ representing $\overline{8}_{21}$

Here $\mathcal{C}_{1}=\{p, r, s, t\}, \mathcal{C}_{0}=\{a, b, c, \bar{a}, \bar{b}, \bar{c}\}$ and $\mathcal{C}_{-1}=\{q\}$, and the nonzero differentials are

$$
\begin{array}{lll}
\partial c=a[\bar{a} \bar{b}] q & \partial \bar{c}=q[b a] \bar{a} & \partial p=[b a][\bar{a} \bar{b}] \\
\partial r=1+[a b c]+a p q & \partial s=[a \bar{a}]+a[\bar{a} \bar{b} \bar{c}]+[c b a] \bar{a} & \partial t=1+[\bar{c} \bar{b} \bar{a}]+q p \bar{a}
\end{array}
$$

where by definition $[x y]=1+x y$. Note that two of the three appearances of $a \bar{a}$ in $\partial s$ can be cancelled since we are working mod 2 . The differential is written in this way in order to facilitate the calculation of augmentations, which amounts to solving four equations over $\mathbb{Z}_{2}$ : the vanishing of $\partial p, \partial r, \partial s$ and $\partial t$.

The solutions to these equations can be described simply by employing the following terminology, which will be used throughout the rest of the paper.

Definition 3.1 The admissible subsets of a triple $x y z$ are $x y z, x y, x, y z$ and $z$. The first two will be referred to as ample, since they contain both $x$ and $y$, and the remaining three will be called sparse. Note that the admissible subsets correspond to solutions to $[x y z]=1$, and the additional constraint $[x y]=1$ selects the sparse ones.

Now consider the two level triples $a b c, \bar{a} \bar{b} \bar{c}$ of degree zero crossings of $K_{2}$. The subsets of these triples that are augmented for a given augmentation $\varepsilon$ will be 
called the levels of $\varepsilon$. Now the vanishing of $\partial r$ and $\partial t$ simply says that both levels of $\varepsilon$ must be admissible, and $\partial p=0$ then forces at least one of these to be ample. The last equation $\partial s=0$ imposes no further restrictions as it is a consequence of the other three equations. It follows that $K_{2}$ has sixteen augmentations, four of which

$$
a b c \bar{a} \bar{b} \bar{c}, a b c \bar{a} \bar{b}, a b \bar{a} \bar{b} \bar{c}, a b \bar{a} \bar{b}
$$

have no sparse levels, while the remaining twelve

$a b c \bar{a}, a b c \bar{b} \bar{c}, a b c \bar{c}, a b \bar{a}, a b \bar{b} \bar{c}, a b \bar{c}, a \bar{a} \bar{b} \bar{c}, a \bar{a} \bar{b}, b c \bar{a} \bar{b} \bar{c}, b c \bar{a} \bar{b}, c \bar{a} \bar{b} \bar{c}, c \bar{a} \bar{b}$

have exactly one. It is now straightforward (although tedious without a computer) to show that the reduced Chekanov polynomial is $t+2$ for the augmentations in the first group, and 1 for those in the second. Alternatively this can be proved using the Duality Theorem (see the last remark in Section 2): It suffices to show that an augmentation is in the first group if and only if the rank $r_{0}$ of the differential $C_{0} \rightarrow C_{-1}$ vanishes, or equivalently $a[\bar{a} \bar{b}]=[b a] \bar{a}=0$. But $a=b=\bar{a}=\bar{b}=1$ is clearly the only solution to these equations. Thus $K_{2}$ has Chekanov number 2 .

Knots with arbitrarily large Chekanov number can now be constructed by taking connected sums of copies of $K_{2}$. To see this, recall that the connected sum $K \# K^{\prime}$ of Legendrian knots can be formed in a variety of ways, all equivalent up to Legendrian isotopy [10]. A convenient one for our purposes is shown in Figure 3

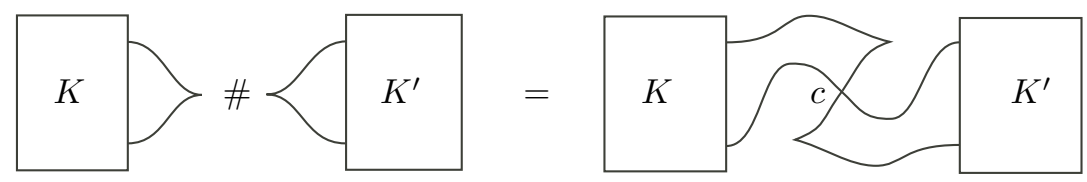

Figure 3: Legendrian connected sum $K \# K^{\prime}$

With this choice, the front of $K \# K^{\prime}$ has one extra degree zero crossing $c$. Its differential graded algebra is generated by $\mathcal{C} \cup \mathcal{C}^{\prime} \cup\{c\}$, with augmentations

$$
\varepsilon \# \varepsilon^{\prime}=\varepsilon \cup \varepsilon^{\prime} \cup\{c\}
$$

where $\varepsilon$ and $\varepsilon^{\prime}$ range over all the augmentations of $K$ and $K^{\prime}$. Fixing such an augmentation yields the chain complex

$$
C \# C^{\prime}=C \oplus C^{\prime} \oplus \mathbb{Z}_{2}
$$

where $C$ and $C^{\prime}$ are the chain complexes for $\varepsilon$ and $\varepsilon^{\prime}$, and the last factor is generated by $c$. The $d$ th differential for $C \# C^{\prime}$ is the block sum of the 


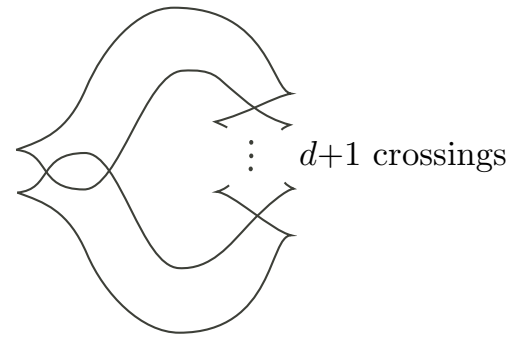

Figure 4: Twist knot $T_{d}$

corresponding differentials for $C$ and $C^{\prime}$, with an extra column of zeros when $d=0$, and an extra nonzero row when $d=1$. Its rank is therefore the sum of the corresponding ranks $r_{d}$ and $r_{d}^{\prime}$, except possibly when $d=1$. In fact in this case the rank is $r_{1}+r_{1}^{\prime}+1$, as can be seen using the duality theorem. It follows that

$$
P_{\varepsilon \# \varepsilon^{\prime}}(t)=P_{\varepsilon}(t)+P_{\varepsilon^{\prime}}(t)-t,
$$

or equivalently, the reduced Chekanov polynomials add $p_{\varepsilon \# \varepsilon^{\prime}}=p_{\varepsilon}+p_{\varepsilon^{\prime}}$. This proves the following (well-known) result, cf [3, Section 12].

Lemma 3.2 The reduced Chekanov polynomials of a Legendrian connected sum are exactly the sums of the reduced Chekanov polynomials of its factors.

Thus the set of reduced Chekanov polynomials of the connected sum $n K_{2}$ of $n$ copies of $K_{2}$ is $\{k t+n+k: k=0, \ldots, n\}$. In particular $\operatorname{ch}\left(n K_{2}\right)=n+1$.

Connected sums can also be used to construct Legendrian knots with prescribed Chekanov polynomials. For example, noting that the twist knot $T_{d}$ in Figure 4 has $t^{d}$ as its unique reduced Chekanov polynomial (as is easily verified), the knot $\#_{d}\left(a_{d} T_{d}\right)$ is seen using the lemma to have (unique) reduced polynomial $\sum_{d} a_{d} t^{d}$. This proves Theorem 1.2 in the Introduction.

One must work a bit harder to produce prime knots with large Chekanov numbers or with prescribed Chekanov polynomials. We show how to do the former, as claimed in Theorem 1.1.

Proof of Theorem 1.1 Let $K_{0}$ be the left-handed trefoil in the tables below (representing $3_{1}$ ). This knot has Chekanov number zero, as can be seen by direct calculation or by appealing to the fact that its rotation number is nonzero (see Proposition [1.3). It is also prime, even in the Legendrian sense (since it has maximal Thurston-Bennequin number). 


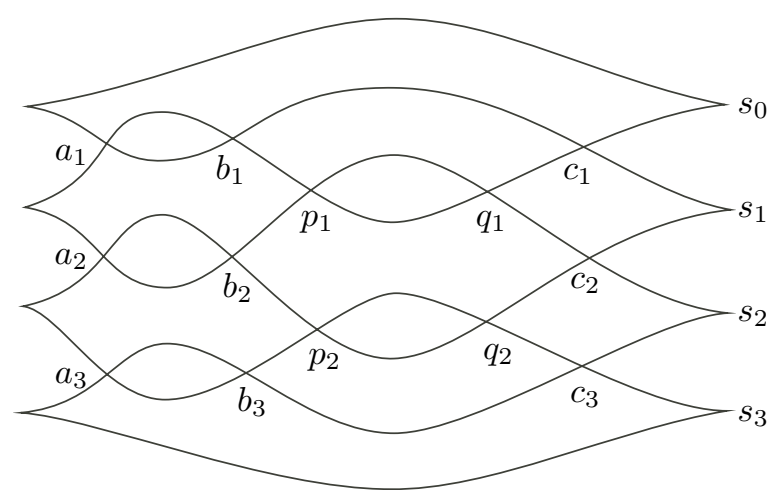

Figure 5: Front for the knot $K_{3}$

It is well-known that the knots $K_{1}$ and $K_{2}$ above, with Chekanov numbers 1 and 2 respectively, are prime. There is a natural way to view them as the first two knots in a sequence $K_{n}$, noting that each can be described as the "plat" closure of a positive braid (see eg [1]) where the plats correspond to cusps in the front: $K_{1}$ is the closure of the 4-braid $\sigma_{2}^{3}$, and $K_{2}$ is the closure of the 6 -braid $\left(\sigma_{2} \sigma_{4}\right)^{2}\left(\sigma_{3}\right)^{2}\left(\sigma_{2} \sigma_{4}\right)$. For $n \geq 3$, let $K_{n}$ be the closure of the $(2 n+2)$-braid $e_{n}^{2} o_{n}^{2} e_{n}$, where $e_{n}=\sigma_{2} \sigma_{4} \ldots \sigma_{2 n}$ and $o_{n}=\sigma_{3} \sigma_{5} \cdots \sigma_{2 n-1}$. The case $n=3$ is shown in Figure 5 .

The labels in Figure [5 suggest a general procedure for labeling $K_{n}$. The degree zero crossings $a_{i}, b_{i}, c_{i}$ occur in descending level triples (one for each $i=1, \ldots, n)$ while the inner crossings $p_{j}, q_{j}$, of degrees \pm 1 respectively, occur in descending level pairs (one for each $j=1, \ldots, n-1$ ). The right cusps are labeled $s_{j}$ for $j=0, \ldots, n$ from top to bottom. Setting variables with subscripts outside their prescribed ranges equal to 1 , the nonzero differentials are

$$
\begin{aligned}
\partial p_{j} & =\left[b_{j} a_{j}\right]\left[a_{j+1} b_{j+1}\right] \\
\partial c_{i} & =q_{i-1}\left[b_{i-1} a_{i-1}\right] a_{i}+a_{i}\left[a_{i+1} b_{i+1}\right] q_{i}+\cdots \\
\partial s_{j} & =\left[a_{j} a_{j+1}\right]+a_{j}\left[a_{j+1} b_{j+1} c_{j+1}\right]+\left[c_{j} b_{j} a_{j}\right] a_{j+1}+\cdots
\end{aligned}
$$

where as above $[x y]=1+x y$ and $[x y z]=x+x y z+z$. Here all monomials with more than one factor of nonzero degree are suppressed, since they never contribute to the differential associated with an augmentation.

Arguing as above it is seen that the augmentations of $K_{n}$ correspond to subsets of the set of degree zero crossings whose levels (ie augmented subsets of the level triples $\left.a_{i} b_{i} c_{i}\right)$ are admissible, and whose sparse levels are nonadjacent. Indeed, the equation $\partial s_{0}=1+\left[a_{1} b_{1} c_{1}\right]+\cdots=0$ establishes the admissibility of the 
top level. Substituting this into $\partial s_{1}=0$ gives $\left[a_{1} a_{2}\right]+a_{1}\left[a_{2} b_{2} c_{2}\right]+a_{2}=0$, which combined with $\partial p_{1}=0$ establishes the admissibility of the second level and forces it to be ample if the top level is sparse. Similarly $\partial s_{2}=\partial p_{2}=0$ then gives the admissibility of the third level, and forces it to be ample if the level above is sparse. Proceeding in this way gives the desired conclusion.

By the last remark in Section 2, the reduced Chekanov polynomial for $\varepsilon \in$ $\operatorname{Aug}\left(K_{n}\right)$ is

$$
p_{\varepsilon}(t)=\left(n-1-r_{0}\right) t+\left(n-r_{0}\right)
$$

where $r_{0}$ is the rank of the associated differential

$$
\partial_{0}: C_{0} \rightarrow C_{-1}=\left\langle q_{1}, \ldots, q_{n-1}\right\rangle .
$$

It is shown below that $r_{0}$ can range from 0 to $\lfloor 2 n / 3\rfloor$, depending on the positions of the sparse levels of $\varepsilon$, and so $\operatorname{ch}\left(K_{n}\right)=\lfloor 2 n / 3\rfloor+1$. In particular

$$
\operatorname{ch}\left(K_{\lfloor 3 k / 2\rfloor-1}\right)=k
$$

for any given integer $k$.

To compute $r_{0}$, observe that it is the rank of the $(n-1) \times n$-matrix $Q$ representing the restriction of $\partial_{0}$ to the subspace $\left\langle c_{1}, \ldots, c_{n}\right\rangle$. Now focus on the $i$ th level for some $i=1, \ldots, n$. The formula for $\partial c_{i}$ above shows that $\partial_{0} c_{i}=q_{i}$ if the level above is sparse, $q_{i-1}$ if the level below is sparse, $q_{i}+q_{i-1}$ if both are sparse, and 0 otherwise. It follows that the nonzero rows of $Q$ are all standard basis vectors for $\mathbb{R}^{n}$, and any particular one $e_{j}$ arises if and only if either level adjacent to the $j$ th one is sparse. Thus $r_{0}$ is just a count of the number of levels adjacent to the sparse levels, and an inductive argument shows that this number assumes all possible values between 0 and $\lfloor 2 n / 3\rfloor$, as claimed.

It remains to show that the knots $K_{n}$ are prime. One way to do this is to use the notion of prime tangles introduced by Kirby and Lickorish in [17. The reader is referred to Lickorish [18, where prime tangles were first used for primality testing in knot theory, for the relevant definitions. The main result of that paper is that any knot that can be written as a sum of two prime tangles is prime, and so it suffices to show that $K_{n}$ is of that form for $n>2$.

It should be noted that in [18, a sum of tangles $S$ and $T$ refers to any link obtained by gluing the tangles together by a homeomorphism of their boundaries, as in Figure 6(a), rather than gluing them together along only part of their boundaries, as in Figure 6(b). The latter produces another tangle called a partial sum (or tangle sum) of $S$ and $T$. To distinguish the two, write $S \oplus T$ for a sum and $S+T$ for a partial sum. 


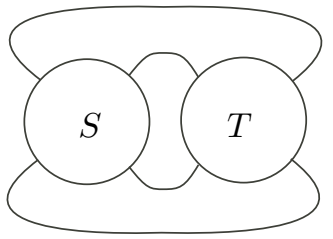

(a) sum

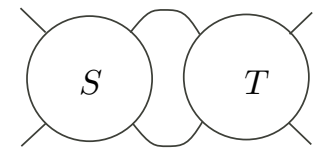

(b) partial sum

Figure 6: Tangle sums

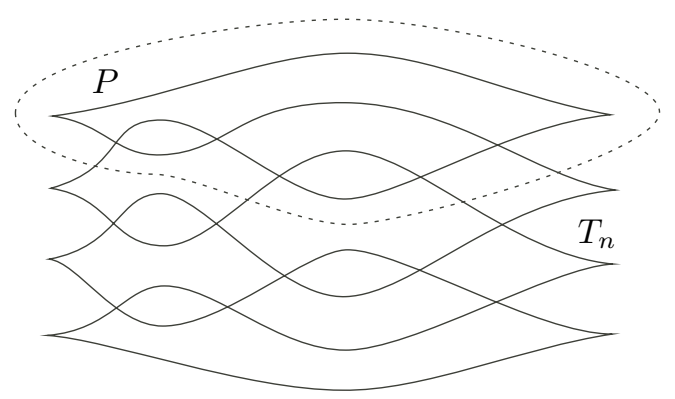

Figure 7: Decomposition of $K_{n}$

Now the decomposition of a knot as a sum of two tangles can be specified by drawing a simple closed curve in a projection plane that intersects the knot in four points, while proper arcs can be used to specify the decomposition of a tangle as a partial sum of tangles. So consider the decomposition $K_{n}=P \oplus T_{n}$ coming from a simple closed curve enclosing the top five crossings in $K_{n}$, as shown in Figure 7 for the case $n=3$.

It is easy to verify that the top tangle $P$, shown in its Legendrian form in Figure 8(a) or its topological equivalent in Figure 8 (b), is prime (see [18, Figure $2 \mathrm{a}])$.

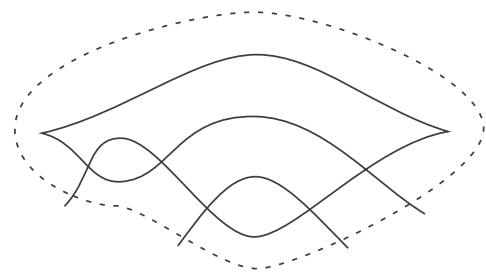

(a) Legendrian form

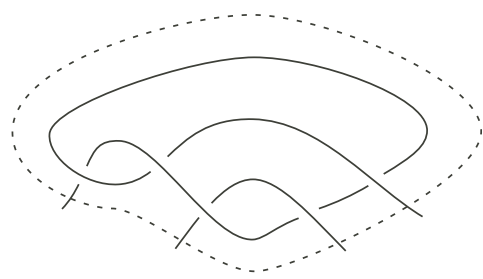

(b) topological form

Figure 8: The prime tangle $P$

The bottom tangle $T_{n}$ is also prime. Indeed it can be written as a partial sum $S_{0}+S_{1}+S_{2}+\cdots$, where $S_{0}$ is the prime tangle (equivalent to $P$ ) encompassing 


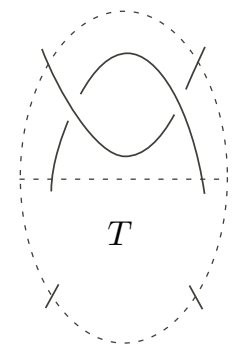

(a) adding a clasp

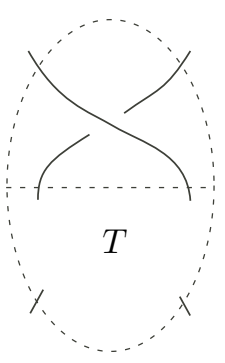

(b) adding a twist

Figure 9: Adding clasps and twists

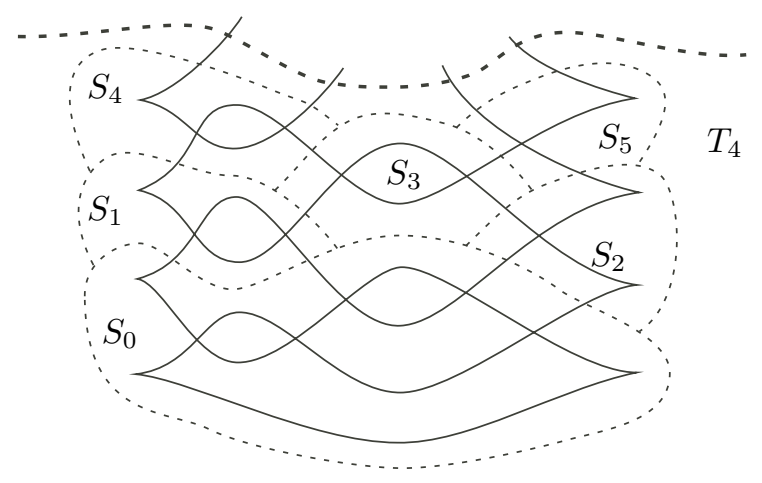

Figure 10: The decomposition of $T_{4}$

the bottom five crossings in $T_{n}$, and the $S_{i}$ are trivial (aka rational) tangles added so as to appear as "clasps" or "twists", as shown in Figure 9.

The case $n=4$ is illustrated in Figure 10.

Since adding a twist does not change the equivalence class of the tangle, and straightforward (innermost disk) arguments show that adding a clasp preserves primality, it follows that $T_{n}$ is prime. Thus for $n>2$, the knot $K_{n}$ is a sum of two prime tangles, and is therefore a prime knot. This completes the proof of Theorem 1.1.

\section{Legendrian tangle replacement}

The purpose of this section is to reinterpret the examples in Section 3 as instances of iterated tangle replacement. In general one can modify a Legendrian knot $K$ that is expressed as a tangle sum $S \oplus T$ by replacing $T$ with another 


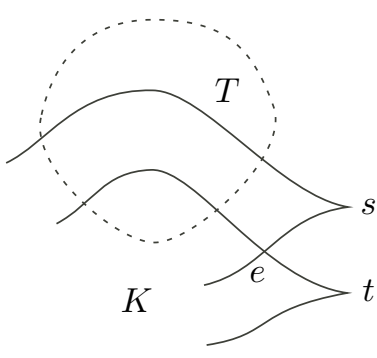

(a) Special Legendrian knot

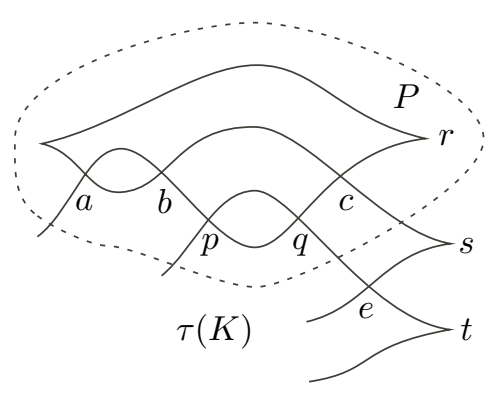

(b) Tangle replacement

Figure 11: Legendrian tangle replacement

tangle $T^{\prime}$, giving $S \oplus T^{\prime}$. For simplicity we study only the case in which $K$ has a simple front with upper right corner as shown in Figure 4(a). Such a knot will be called a special Legendrian knot. Also assume that $T$ is the trivial tangle near the top right cusp $s$, and that $T^{\prime}$ is the prime tangle $P$ considered in the last section. The Legendrian knot resulting from this particular tangle replacement will be denoted $\tau(K)$.

Remark 4.1 If $S$ is a prime tangle, then the knot $\tau(K)=S \oplus P$ is prime, by Lickorish's theorem [18. In fact all the knots in the sequence

$$
\tau(K), \tau^{2}(K), \tau^{3}(K), \ldots
$$

are then prime. This can be seen by induction since $S \oplus P$ can be written as $S^{\prime} \oplus T$ where $S^{\prime}$ is a prime tangle obtained by adding two "clasps" and one "twist" to $S$ as in Section 3 .

Theorem 4.2 If $K$ is any special Legendrian knot with nonzero Chekanov number, then the sequence of Chekanov numbers

$$
\operatorname{ch}(\tau(K)), \operatorname{ch}\left(\tau^{2}(K)\right), \operatorname{ch}\left(\tau^{3}(K)\right), \ldots
$$

grows without bound.

For example, applying the theorem to the mirror trefoil $\left(\overline{3}_{1}\right.$ in the table below) recovers the unboundedness of the Chekanov numbers of the knots $\overline{8}_{21}, \ldots$ discussed in Section 3, and the fact that these knots are prime follows from Remark 4.1] The mirror figure eight $\left(\overline{4}_{1}\right.$ in the table) produces another sequence $\overline{9}_{45}, \ldots$ of prime knots with increasing Chekanov numbers. (It is suggestive that $\overline{8}_{21}$ and $\overline{9}_{45}$ are the only knots in the table below with Chekanov number greater than one.) In fact, every knot with nonzero Chekanov number is isotopic to a special Legendrian knot, and thence yields such a sequence. 


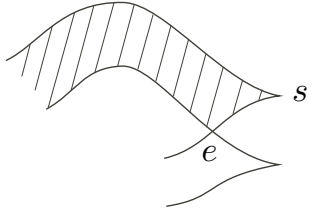

(a) disks for $s_{1}$

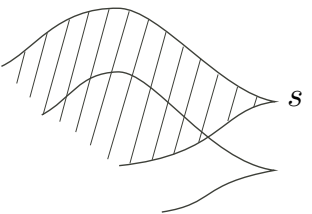

(b) disks for $s_{2}$

Figure 12: Decomposition of $\partial s$

The theorem will be proved by analyzing the effect of a tangle replacement on the the set of Chekanov polynomials of $K$. This analysis depends on a number of factors, the most basic of which is the difference between the Maslov potentials of the upper and lower strands of $T$. (See Section 2 for the definition of the Maslov potential.) This potential difference will be denoted by $d$ below, and will be referred to as the Maslov number of $K$.

Note that the Maslov number of $\tau^{n}(K)$ is equal to 1 for each $n>0$. Thus for the purposes of proving the theorem, it suffices to consider the case $d=1$ once it is shown in general that $\operatorname{ch}(\tau(K)) \neq 0$ (which follows from Lemma 4.4 when $d \neq 0$ and Remark 4.5 when $d=0)$. But for other purposes it may be of interest to study the general case. Hence we do not at present put any restrictions on $d$.

The basic strategy of the proof is to identify a class of augmentations of $K$ that are "fertile" enough to cause the Chekanov number to grow under iterated tangle replacements. Writing $(\mathcal{A}, \partial)$ for the differential graded algebra of $K$, consider the elements $s_{1}$ and $s_{2}$ in $\mathcal{A}$ defined by

$$
\partial s=1+s_{1} e+s_{2} .
$$

Thus $s_{1} e$ is the sum of all the monomials $\partial D$ associated with admissible disks $D$ (for $s$ ) that end in the crossing $e$, while $s_{2}$ is the sum of the remaining admissible disk monomials (see Figure 12). Note that if $d \neq 1$, then the term $s_{1} e$ can be ignored for the purposes of computing Chekanov polynomials, since $e$ and $s_{1}$ are then both of nonzero degree (their degrees add to zero and $|e|=$ $1-d \neq 0)$.

Definition 4.3 An augmentation $\varepsilon$ of $K$, viewed as a subset of the degree zero crossings, is fertile if either (a) $d>1$, or (b) $d=1, s_{1}=0$ (in $\mathbb{Z}_{2}$, after substituting 1 for the crossings in $\varepsilon$ and 0 for the remaining generators of $\mathcal{A}$ ), and $\partial_{0} e$ is a sum of differentials of some of the other degree zero crossings of $K \dagger$

${ }^{\dagger}$ Here $\partial_{0}$ is the differential $C_{0} \rightarrow C_{-1}$ in the chain complex defined by $\varepsilon$. 
Now what happens after the tangle replacement? There are five new crossings $a, b, c, p, q$ and one new right cusp $r$ (see Figure 4). The remaining crossings and cusps in $\tau(K)$ retain their labels from $K$. At this point it is convenient to impose a restriction on $d$, which will remain in effect for most of what follows, with the exception of Remark 4.5 .

Assumption The Maslov number $d$ of $K$ is nonzero.

Thus the crossings $a, b, c$, which are all of degree zero, are the only new crossings that can potentially be augmented. The crossings $p$ and $q$ are of nonzero degrees $\pm d$, respectively.

Writing $\left(\mathcal{A}^{\tau}, \partial^{\tau}\right)$ for the differential graded algebra of $\tau(K)$ it is clear that $\partial^{\tau} x=\partial x$ for all the generators (crossings or right cusps) $x$ of $\mathcal{A}$ except $x=e$, $s$ and $t$. For these exceptional cases, an inspection of Figure 4 shows that

$$
\begin{aligned}
\partial^{\tau} e & =\partial e+q[b a] s_{2}+\cdots \\
\partial^{\tau} s & =1+a(\partial s-1)+c[b a] s_{2}+\cdots \\
\partial^{\tau} t & =\partial t+\cdots
\end{aligned}
$$

where $[b a]=1+b a$, and monomials with more than one factor of nonzero degree are omitted since they do not contribute to the differential for any augmentation of $\tau(K)$. Similarly the nonzero differentials of the new generators of $\mathcal{A}^{\tau}$ are

$$
\begin{aligned}
\partial^{\tau} p & =[b a] s_{1} \\
\partial^{\tau} c & =a s_{1} q \\
\partial^{\tau} r & =1+[a b c]+\cdots
\end{aligned}
$$

where $[a b c]=a+a b c+c$.

Lemma 4.4 If $d \neq 0$, then the augmentations of $\tau(K)$ are exactly the sets $\varepsilon \cup \alpha$ where $\varepsilon$ is an augmentation of $K$ and $\alpha$ is an admissible subset of abc, with the following restriction when $d=1$ :

$$
s_{1}=1 \text { (in } \mathbb{Z}_{2} \text {, after the usual substitution) } \Longrightarrow \alpha \text { is ample }
$$

(Recall from Section 3 that the admissible subsets of $a b c$ are $a b c, a b, a, b c, c$, of which the first two are ample and the last three are sparse.)

Proof Start with an augmentation $\varepsilon^{\tau}$ of $\tau(K)$, written as the union $\varepsilon \cup \alpha$ where $\alpha=\varepsilon^{\tau} \cap a b c$. If $\varepsilon$ were not an augmentation of $K$, then there would exist a crossing $x$ in $K$ for which $\partial x=1$ (after substitution). Since $\partial^{\tau} x=0$ (as $\varepsilon^{\tau}$ is an augmentation) it would follow that $x=s$, and so $\partial^{\tau} s=1+c[b a] s_{2}=0$. 
This would imply $c=[b a]=s_{2}=1$, which in turn would force $s_{1}=1$, since $\partial s=1$, and thence contradict $\partial^{\tau} p=0$. Thus $\varepsilon$ is an augmentation of $K$.

Now the condition $\partial^{\tau} r=0$ (after substitution) is equivalent to the admissibility of $\alpha$, and the condition $\partial^{\tau} p=0$ imposes the desired restriction $(\star)$ on $\alpha$ since $[b a]$ vanishes if and only if $\alpha$ is ample. (Note that this restriction only arises when $d=1$ since $s_{1}=0$ otherwise.) The remaining conditions $\partial^{\tau} s=0$ and $\partial^{\tau} t=0$ impose no further restrictions on $\alpha$. This is clear for the latter, and for the former it follows from the observation that $\partial s=0$ (since $\varepsilon$ is an augmentation) which shows that $\partial^{\tau} s=1+a+c[b a] s_{2}=0$ is equivalent to the pair of implications $s_{2}=1 \Longrightarrow 1+a+c[b a]=1+[a b c]=0$ (which is the admissibility of $\alpha$ ) and $s_{2}=0 \Longrightarrow a=1$ (which follows from $(\star)$ since $\partial s=0$ shows that $s_{1}$ and $s_{2}$ cannot simultaneously vanish).

Remark 4.5 If $d=0$, then the same argument shows that the augmentations of $\tau(K)$ that do not contain $p$ or $q$ are exactly the sets $\varepsilon \cup \alpha$, where $\varepsilon$ is an augmentation of $K$ and $\alpha$ is an admissible subset of $a b c$. However there may also exist augmentations that include one or both of $p$ and $q$.

Lemma 4.6 If $d \neq 0$, then the reduced Chekanov polynomial of $\tau(K)$ corresponding to an augmentation $\varepsilon \cup \alpha$ as above is of the form $p_{\varepsilon \cup \alpha}(t)=p_{\varepsilon}(t)+\Delta(t)$, where

$$
\Delta(t)= \begin{cases}1+t^{d} & \text { for } \alpha \text { ample } \\ 1-t^{d-1} & \text { for } \alpha \text { sparse }\end{cases}
$$

if $\varepsilon$ is fertile. If $\varepsilon$ is infertile then $\Delta(t)=1+t^{|d|}$ or 0 according to whether $s_{1}=0$ or 1 . (Note that $d<0 \Longrightarrow s_{1}=0$.)

Proof We must analyze how the chain complexes $(C, \partial)$ and $\left(C^{\tau}, \partial^{\tau}\right)$ for $K$ and $\tau(K)$, associated with the augmentations $\varepsilon$ and $\varepsilon \cup \alpha$, are related. As noted above, there are six new generators in $C^{\tau}$, namely $a, b, c \in C_{0}^{\tau}, r \in C_{1}^{\tau}$, $p \in C_{d}^{\tau}$ and $q \in C_{-d}^{\tau}$. Write $n_{k}$ and $n_{k}^{\tau}$ for the dimensions of $C_{k}$ and $C_{k}^{\tau}$, and $r_{k}$ and $r_{k}^{\tau}$ for the ranks of $\partial_{k}$ and $\partial_{k}^{\tau}$. It follows from the last remark in Section 2 that the coefficient $\Delta_{k}$ of $t^{k}$ in the difference $\Delta(t)=p_{\varepsilon \cup \alpha}(t)-p_{\varepsilon}(t)$ of the reduced Chekanov polynomials is

$$
\Delta_{k}= \begin{cases}\Delta n_{-k}-\Delta r_{-k+1}-\Delta r_{-k-1} & \text { for } k>0 \\ 1+(-1)^{d}-\sum_{i>0}(-1)^{i} \Delta_{i} & \text { for } k=0\end{cases}
$$

where $\Delta n_{k}=n_{k}^{\tau}-n_{k}$ and $\Delta r_{k}=r_{k}^{\tau}-r_{k}$. Thus it suffices to compute $\Delta n_{j}$ and $\Delta r_{j}$ for all $j \leq 0$. Evidently $\Delta n_{0}=3, \Delta n_{-|d|}=1$ and $\Delta n_{j}=0$ for all 
other $j<0$. To compute $\Delta r_{j}$ it is convenient to consider the cases $d \neq 1$ and $d=1$ separately.

First assume $d \neq 1$. Then working in $\mathbb{Z}_{2}$ after the usual substitution, $\partial_{0}^{\tau} c=0$, since the degrees of $s_{1}$ and $q$ are both nonzero, and so $\Delta r_{0}=0$. It is clear that $\Delta r_{j}=0$ for all $j<0$, except possibly $j=1-d$ with $d>1$. (Note that $\partial_{-|d|}^{\tau} p=0$ if $d<0$ since $s_{1}=0$.) But in this case $\partial_{1-d}^{\tau} e=\partial_{1-d} e+q[b a] s_{2}$ and so

$$
\Delta r_{1-d}=[b a] s_{2} .
$$

Note that $[b a]=0 \Longleftrightarrow \alpha$ is ample, and $s_{2}=0 \Longrightarrow s_{1}=1 \Longrightarrow \alpha$ is ample (by $(\star)$ ), whence $\Delta r_{1-d}=0 \Longleftrightarrow \alpha$ is ample. The formula for $\Delta(t)$ follows readily.

Now assume $d=1$. Then $\Delta r_{j}=0$ for all $j<0$, and so the formulas above show that $\Delta(t)=1+t$ or 0 according to whether $\Delta r_{0}=0$ or 1 . To compute $\Delta r_{0}$, note that (the matrix for) $\partial_{0}^{\tau}$ is obtained from $\partial_{0}$ by adding three new zero columns, corresponding to the generators $a, b$ and $c$, and then adding a new row, corresponding to $q$. All the entries in this last row are zero except possibly the ones in the $e$ and $c$ columns, denoted $\langle q \mid e\rangle$ and $\langle q \mid c\rangle$. In particular (working in $\mathbb{Z}_{2}$ )

$$
\langle q \mid e\rangle=[b a] s_{2} \quad \text { and } \quad\langle q \mid c\rangle=a s_{1}
$$

and so $\langle q \mid e\rangle=0 \Longleftrightarrow \alpha$ is ample (as shown above) and $\langle q \mid c\rangle=0 \Longleftrightarrow s_{1}=0$ (since $a=0 \Longrightarrow s_{1}=0$ by $(\star)$ ). Therefore, if $\langle q \mid c\rangle=0$ (ie $s_{1}=0$ ) then $\Delta r_{0}=0$ if and only if either $\langle q \mid e\rangle=0$ (ie $\alpha$ is ample), or $\langle q \mid e\rangle=1$ and the $e$ column in $\partial_{0}$ is independent of the other columns (ie $\alpha$ is sparse and $\varepsilon$ is infertile). If $\langle q \mid c\rangle=1$ (ie $s_{1}=1$ ) then clearly $\Delta r_{0}=1$. Unravelling the definitions now gives the desired result.

All the ingredients are now in place to prove the theorem.

Proof of Theorem 4.2 Fix an augmentation $\varepsilon$ of $K$, and consider all words $w$ in the letters $a$ (for ample) and $s$ (for sparse) satisfying

(1) $w$ begins with $a$, and

(2) $s$ never appears twice in a row in $w$.

Any such $w$ naturally specifies a family of augmentations of $\tau^{n}(K)$, where $n$ is the length of $w$, as follows:

Associate the one letter word $a$ to the family $\{\varepsilon \cup a b c, \varepsilon \cup a b\}$ of augmentations of $\tau(K)$ obtained by extending $\varepsilon$ by an ample subset of $a b c$. (Lemma 4.4 and 
Remark 4.5 show that these are indeed augmentations.) Now $\tau(K)$ has Maslov degree 1 , and it is easily verified that the new $s_{1}$ (the one for $\tau(K)$ with either augmentation in $a$ ) is zero. Therefore the augmentations in $a$ can be extended to augmentations of $\tau^{2}(K)$ either amply (specified by $a a$ ) or sparsely (specified by as).

Inductively, if $w$ specifies a family of augmentations of $\tau^{n}(K)$ for some $n>1$, then the word $w a$ specifies the ample extensions of $w$ to augmentations of $\tau^{n+1}(K)$, while $w s$ specifies the sparse extensions. To see that condition (2) above is necessary, note that the $s_{1}$ for any augmentation $\varepsilon^{\prime}$ in $w$ is zero if and only if $w$ ends in $a$, and apply condition $(\star)$ in Lemma 4.4

Also, noting that an $\varepsilon^{\prime}$ in $w$ is fertile if and only if $w$ ends in $a a$, it follows from Lemma 4.6 that the reduced Chekanov polynomials for the augmentations associated with $w$ are all equal. This polynomial will be denoted by $p_{w}(t)$.

To complete the proof it suffices for each $m>1$ to produce a collection of $m$ words of equal length whose polynomials are distinct. For example, the polynomials associated with the words $w_{k}=\left(a^{2}\right)^{k}(s a)^{m-k+1}$ (for $\left.k=1, \ldots, m\right)$ satisfy

$$
p_{w_{k}}(t)-p_{a^{2}}(t)=(m+k-2)(1+t)
$$

by direct calculation using Lemma 4.6. Thus these $m$ words, all of length $2 m+2$, have distinct polynomials, and the theorem is proved.

Remark 4.7 The method used in this proof shows how to compute the full list of Chekanov polynomials of $\tau^{n}(K)$ for each $n>0$, if $K$ is a special Legendrian knot with nonzero Maslov number $d$ whose Chekanov polynomials are known.

The discussion above suggests two directions for further investigation:

Problem 4.8 (a) Analyze the effect of a special tangle replacement on the Chekanov polynomials (as in Lemma 4.6) for knots of Maslov number zero.

(b) Investigate the effects of arbitrary tangle replacements, at different locations and using prime tangles other than $P$.

\section{Tabulation}

The following is a list of maxima $\oplus$ Legendrian fronts for each knot of nine or fewer crossings. The knot types are ordered as in the original tabulation

\footnotetext{
†This means 'of maximal Thurston-Bennequin number in their knot type'.
} 
of Alexander and Briggs: $n_{k}$ is the $k$ th $n$-crossing knot. Taking the ambient orientation into account, each chiral knot in the Alexander-Briggs table actually corresponds to a pair of knot types (up to topological isotopy) that are mirror images of each other. These are distinguished by writing $n_{k}$ for the knot that appears in Rolfsen's table [22], and $\bar{n}_{k}$ for its mirror image. The fronts for these "mirror pairs" occur side by side in the list below, with $n_{k}$ appearing first. The other columns in the table give the classical invariants $(t b, r)$ and the reduced Chekanov polynomials of the knots and their mirrors.

The achiral knots in the table are identified with an asterisk. For these knots, the mirror pairs $n_{k}, \bar{n}_{k}$ are topologically isotopic, although they need not be Legendrian isotopic. For example $4_{1}, \overline{4}_{1}$ are Legendrian isotopic by [9], while the pairs $8_{3}, \overline{8}_{3}$ and $8_{12}, \overline{8}_{12}$ are not, since they are distinguished by their Chekanov polynomials. The analogous question for the remaining achiral pairs in the table is left to the reader.

A special feature of the particular fronts shown below is that the mirror pairs are "rotationally related". More precisely, the front for $\bar{n}_{k}$ is obtained by rotating the front for $n_{k}$ a quarter turn clockwise, and then swapping cusps for caps. Another special feature is that all the fronts are of minimal crossing number, with the exception of $9_{42}$ and its mirror (for which we have not been able to find minimal crossing, rotationally related fronts).

Our indebtedness to the tabulation efforts of Yufa [26] and Branson [2] (who first investigated the possibility of finding rotationally related fronts for chiral pairs) is evident. Most of the fronts drawn below appear in one or both of their tables. To verify that all the knots shown are maximal (with the possible exception of $\overline{9}_{42}$ ) we appeal to Ng's computation of the maximal ThurstonBennequin numbers for knots up to nine crossings [19, which builds on Yufa's tabulation. The classical invariants and Chekanov polynomials for the listed knots were computed using a Mathematica program written by the first author. 
Table 1: Invariants of Legendrian Knots

\begin{tabular}{|c|c|c|c|c|c|}
\hline $\begin{array}{l}\text { knot } \\
\text { type }\end{array}$ & $(t b, r)$ & $\begin{array}{c}\text { reduced } \\
\text { polynomials }\end{array}$ & front and mirror & $\begin{array}{c}\text { reduced } \\
\text { polynomials }\end{array}$ & $(t b, r)$ \\
\hline $0_{1}^{*}$ & $(-1,0)$ & 0 & & 0 & $(-1,0)$ \\
\hline $3_{1}$ & $(-6,1)$ & & & 1 & $(1,0)$ \\
\hline $4_{1}^{*}$ & $(-3,0)$ & $t$ & & $t$ & $(-3,0)$ \\
\hline $5_{1}$ & $(-10,3)$ & & & 2 & $(3,0)$ \\
\hline $5_{2}$ & $(-8,1)$ & & & 1 & $(1,0)$ \\
\hline $6_{1}$ & $(-5,0)$ & $2 t$ & & $t$ & $(-3,0)$ \\
\hline $6_{2}$ & $(-7,0)$ & & & $1+t$ & $(-1,0)$ \\
\hline $6_{3}^{*}$ & $(-4,1)$ & & & & $(-4,1)$ \\
\hline $7_{1}$ & $(-14,5)$ & & & 3 & $(5,0)$ \\
\hline
\end{tabular}




\begin{tabular}{|c|c|c|c|c|c|}
\hline $\begin{array}{l}\text { knot } \\
\text { type }\end{array}$ & $(t b, r)$ & $\begin{array}{c}\text { reduced } \\
\text { polynomials }\end{array}$ & front and mirror & $\begin{array}{c}\text { reduced } \\
\text { polynomials }\end{array}$ & $(t b, r)$ \\
\hline $7_{2}$ & $(-10,1)$ & & & 1 & $(1,0)$ \\
\hline $7_{3}$ & $(3,0)$ & $2 t^{2}$ & & & $(-12,1)$ \\
\hline $7_{4}$ & $(1,0)$ & 1 & & & $(-10,1)$ \\
\hline $7_{5}$ & $(-12,1)$ & & & $1+t^{2}$ & $(3,0)$ \\
\hline $7_{6}$ & $(-8,1)$ & & & $t+t^{2}$ & $(-1,0)$ \\
\hline $7_{7}$ & $(-4,1)$ & & & $2 t$ & $(-5,0)$ \\
\hline $8_{1}$ & $(-7,0)$ & $3 t$ & & $t^{5}$ & $(-3,0)$ \\
\hline $8_{2}$ & $(-11,4)$ & & & $2+t$ & $(1,0)$ \\
\hline $8_{3}^{*}$ & $(-5,0)$ & $2 t^{3}$ & & $2 t$ & $(-5,0)$ \\
\hline 84 & $(-7,2)$ & & & $1+2 t$ & $(-3,0)$ \\
\hline $8_{5}$ & $(1,0)$ & $2+t$ & & & $(-11,0)$ \\
\hline
\end{tabular}




\begin{tabular}{|c|c|c|c|c|c|}
\hline $\begin{array}{l}\text { knot } \\
\text { type }\end{array}$ & $(t b, r)$ & $\begin{array}{c}\text { reduced } \\
\text { polynomials }\end{array}$ & front and mirror & $\begin{array}{c}\text { reduced } \\
\text { polynomials }\end{array}$ & $(t b, r)$ \\
\hline $8_{6}$ & $(-9,2)$ & & & $1+t^{3}$ & $(-1,0)$ \\
\hline $8_{7}$ & $(-2,1)$ & & & & $(-8,1)$ \\
\hline $8_{8}$ & $(-4,1)$ & & & & $(-6,1)$ \\
\hline $8_{9}^{*}$ & $(-5,2)$ & & & & $(-5,2)$ \\
\hline $8_{10}$ & $(-2,1)$ & & & & $(-8,1)$ \\
\hline $8_{11}$ & $(-9,0)$ & & & $t+t^{2}$ & $(-1,0)$ \\
\hline $8_{12}^{*}$ & $(-5,0)$ & $t+t^{3}$ & & $2 t$ & $(-5,0)$ \\
\hline $8_{13}$ & $(-4,1)$ & & & & $(-6,1)$ \\
\hline $8_{14}$ & $(-9,2)$ & & & $1+t$ & $(-1,0)$ \\
\hline $8_{15}$ & $(-13,0)$ & & & $1+t^{2}$ & $(3,0)$ \\
\hline
\end{tabular}




\begin{tabular}{|c|c|c|c|c|c|}
\hline $\begin{array}{l}\text { knot } \\
\text { type }\end{array}$ & $(t b, r)$ & $\begin{array}{c}\text { reduced } \\
\text { polynomials }\end{array}$ & front and mirror & $\begin{array}{c}\text { reduced } \\
\text { polynomials }\end{array}$ & $(t b, r)$ \\
\hline $8_{16}$ & $(-8,1)$ & & & & $(-2,1)$ \\
\hline $8_{17}^{*}$ & $(-5,0)$ & & & & $(-5,0)$ \\
\hline $8_{18}^{*}$ & $(-5,0)$ & & & & $(-5,0)$ \\
\hline $8_{19}$ & $(5,0)$ & 3 & & & $(-12,1)$ \\
\hline $8_{20}$ & $(-6,1)$ & & & & $(-2,1)$ \\
\hline $8_{21}$ & $(-9,0)$ & & & $\begin{array}{c}1 \\
2+t\end{array}$ & $(1,0)$ \\
\hline $9_{1}$ & $(-18,7)$ & & & 4 & $(7,0)$ \\
\hline $9_{2}$ & $(-12,1)$ & & & $t^{6}$ & $(1,0)$ \\
\hline $9_{3}$ & $(5,0)$ & $3 t^{2}$ & & & $(-16,5)$ \\
\hline $9_{4}$ & $(-14,3)$ & & & $2 t^{4}$ & $(3,0)$ \\
\hline
\end{tabular}




\begin{tabular}{|c|c|c|c|c|c|}
\hline $\begin{array}{l}\text { knot } \\
\text { type }\end{array}$ & $(t b, r)$ & $\begin{array}{c}\text { reduced } \\
\text { polynomials }\end{array}$ & front and mirror & $\begin{array}{c}\text { reduced } \\
\text { polynomials }\end{array}$ & $(t b, r)$ \\
\hline $9_{5}$ & $(1,0)$ & & & & $(-12,1)$ \\
\hline $9_{6}$ & $(-16,3)$ & & & $2+t^{2}$ & $(5,0)$ \\
\hline $9_{7}$ & $(-14,1)$ & & & $1+t^{4}$ & $(3,0)$ \\
\hline $9_{8}$ & $(-8,1)$ & & & $1+2 t$ & $(-3,0)$ \\
\hline $9_{9}$ & $(-16,1)$ & & & $1+2 t^{2}$ & $(5,0)$ \\
\hline $9_{10}$ & $(3,0)$ & & & & $(-14,3)$ \\
\hline $9_{11}$ & $(1,0)$ & $t+2 t^{2}$ & & & $(-12,1)$ \\
\hline $9_{12}$ & $(-10,1)$ & & & $t^{3}+t^{4}$ & $(-1,0)$ \\
\hline $9_{13}$ & $(3,0)$ & & & & $(-14,3)$ \\
\hline $9_{14}$ & $(-4,1)$ & & & $3 t$ & $(-7,0)$ \\
\hline
\end{tabular}




\begin{tabular}{|c|c|c|c|c|c|}
\hline $\begin{array}{l}\text { knot } \\
\text { type }\end{array}$ & $(t b, r)$ & $\begin{array}{c}\text { reduced } \\
\text { polynomials }\end{array}$ & front and mirror & $\begin{array}{c}\text { reduced } \\
\text { polynomials }\end{array}$ & $(t b, r)$ \\
\hline $9_{15}$ & $(-1,0)$ & $t+t^{2}$ & & & $(-10,1)$ \\
\hline $9_{16}$ & $(5,0)$ & 3 & & & $(-16,1)$ \\
\hline $9_{17}$ & $(-8,3)$ & & & $1+2 t$ & $(-3,0)$ \\
\hline $9_{18}$ & $(-14,1)$ & & & $1+t^{2}$ & $(3,0)$ \\
\hline $9_{19}$ & $(-6,1)$ & & & $2 t$ & $(-5,0)$ \\
\hline $9_{20}$ & $(-12,1)$ & & & $2+t$ & $(1,0)$ \\
\hline $9_{21}$ & $(-1,0)$ & & & & $(-10,1)$ \\
\hline $9_{22}$ & $(-3,0)$ & $1+2 t$ & & & $(-8,1)$ \\
\hline $9_{23}$ & $(-14,1)$ & & & $1+t^{2}$ & $(3,0)$ \\
\hline $9_{24}$ & $(-6,1)$ & & & & $(-5,2)$ \\
\hline
\end{tabular}




\begin{tabular}{|c|c|c|c|c|c|}
\hline $\begin{array}{l}\text { knot } \\
\text { type }\end{array}$ & $(t b, r)$ & $\begin{array}{c}\text { reduced } \\
\text { polynomials }\end{array}$ & front and mirror & $\begin{array}{c}\text { reduced } \\
\text { polynomials }\end{array}$ & $(t b, r)$ \\
\hline $9_{25}$ & $(-10,1)$ & & & $1+t^{3}$ & $(-1,0)$ \\
\hline $9_{26}$ & $(-2,1)$ & & & & $(-9,0)$ \\
\hline $9_{27}$ & $(-6,1)$ & & & & $(-5,2)$ \\
\hline $9_{28}$ & $(-9,0)$ & & & & $(-2,1)$ \\
\hline $9_{29}$ & $(-8,3)$ & & & $1+2 t$ & $(-3,0)$ \\
\hline $9_{30}$ & $(-6,1)$ & & & & $(-5,0)$ \\
\hline $9_{31}$ & $(-9,2)$ & & & & $(-2,1)$ \\
\hline $9_{32}$ & $(-2,1)$ & & & & $(-9,2)$ \\
\hline $9_{33}$ & $(-6,1)$ & & & & $(-5,2)$ \\
\hline $9_{34}$ & $(-6,1)$ & & & & $(-5,0)$ \\
\hline
\end{tabular}




\begin{tabular}{|c|c|c|c|c|c|}
\hline $\begin{array}{l}\text { knot } \\
\text { type }\end{array}$ & $(t b, r)$ & $\begin{array}{c}\text { reduced } \\
\text { polynomials }\end{array}$ & front and mirror & $\begin{array}{c}\text { reduced } \\
\text { polynomials }\end{array}$ & $(t b, r)$ \\
\hline $9_{35}$ & $(-12,1)$ & & & 1 & $(1,0)$ \\
\hline $9_{36}$ & $(1,0)$ & $1+t+t^{2}$ & & & $(-12,1)$ \\
\hline $9_{37}$ & $(-6,1)$ & & & $2 t^{3}$ & $(-5,0)$ \\
\hline $9_{38}$ & $(-14,1)$ & & & $1+t^{2}$ & $(3,0)$ \\
\hline $9_{39}$ & $(-1,0)$ & $1+t$ & & & $(-10,1)$ \\
\hline $9_{40}$ & $(-9,2)$ & & & & $(-2,1)$ \\
\hline $9_{41}$ & $(-7,0)$ & $3 t$ & & & $(-4,1)$ \\
\hline $9_{42}$ & $(-3,0)$ & $1+2 t$ & & & $(-5,0)$ \\
\hline $9_{43}$ & $(1,0)$ & $2+t$ & & & $(-10,1)$ \\
\hline $9_{44}$ & $(-6,1)$ & & & $t$ & $(-3,0)$ \\
\hline $9_{45}$ & $(-10,1)$ & & & $\begin{array}{c}1 \\
1+t+t^{2}\end{array}$ & $(1,0)$ \\
\hline
\end{tabular}




\begin{tabular}{|c|c|c|c|c|c|}
\hline $\begin{array}{l}\text { knot } \\
\text { type }\end{array}$ & $(t b, r)$ & $\begin{array}{c}\text { reduced } \\
\text { polynomials }\end{array}$ & front and mirror & $\begin{array}{c}\text { reduced } \\
\text { polynomials }\end{array}$ & $(t b, r)$ \\
\hline $9_{46}$ & $(-1,0)$ & 0 & & $3 t$ & $(-7,0)$ \\
\hline $9_{47}$ & $(-2,1)$ & & & $3 t$ & $(-7,0)$ \\
\hline $9_{48}$ & $(-1,0)$ & $1+t$ & & & $(-8,1)$ \\
\hline $9_{49}$ & $(3,0)$ & 2 & & & $(-12,1)$ \\
\hline
\end{tabular}

\section{References}

[1] J S Birman, Braids, Links, and Mapping Class Groups, Annals of Math. Studies 82 Princeton University Press, Princeton NJ (1974) MathReview

[2] M Branson, Tabulation of the Legendrian knots by ambient isotopy, preprint (2001)

[3] Yu V Chekanov, Differential algebra of Legendrian links, Inventiones Math. 150 (2002) 441-483 MathReview

[4] Yu V Chekanov, Invariants of Legendrian knots, from: "Proceedings of the International Congress of Mathematicians, Volume II (Beijing 2002)", Higher Ed. Press, Beijing (2002) 385-394 MathReview

[5] Yu V Chekanov, P E Pushkar, Combinatorics of fronts of Legendrian links and the Arnol'd 4-conjectures, Uspekhi Mat. Nauk 60 (2005), 1(361) 99-154; translated in Russian Math. Surveys 60 (2005) 95-149 MathReview

[6] Ya Eliashberg, Invariants in contact topology, from: "Proceedings of the International Congress of Mathematicians, Volume II (Berlin 1998)" Doc. Math. (1998) 327-338 MathReview

[7] Ya Eliashberg, M Fraser, Classification of topologically trivial Legendrian knots, from "Geometry, Topology, and Dynamics (Montreal, PQ, 1995)", CRM Proc. Lecture Notes 15, AMS, Providence, RI, USA (1998) 17-51 MathReview 
[8] Ya Eliashberg, A Givental, H Hofer, Introduction to symplectic field theory, from: "GAFA 2000 (Tel Aviv, 1999)" Geom. Funct. Anal., Special volume, Part II (2000) 560-673 MathReview

[9] J Etnyre, K Honda, Knots and contact geometry I: torus knots and the figure eight knot, J. Sympl. Geom. 1 (2001) 63-120 MathReview

[10] J Etnyre, K Honda, On connected sums and Legendrian knots, Adv. Math. 179 (2003) 59-74 MathReview

[11] J Etnyre, K Honda, Cabling and transverse simplicity, to appear in Annals of Math. arXiv:math.SG/0306330

[12] J Etnyre, L Ng, Problems in low dimensional contact topology, from: "Topology and geometry of manifolds (Athens, GA, 2001)" Proc. Sympos. Pure Math. 71 (2003) 337-357 MathReview

[13] J Etnyre, L Ng, J Sabloff, Invariants of Legendrian knots and coherent orientations, J. Sympl. Geom. 1 (2002) 321-367 MathReview

[14] D Fuchs, Chekanov-Eliashberg invariant of Legendrian knots: existence of augmentations, J. Geom. and Physics 47 (2003) 43-65 MathReview

[15] D Fuchs, T Ishkhanov, Invariants of Legendrian knots and decompostions of front diagrams, Moscow Math. J. 4 (2004) 707-717, 783 MathReview

[16] H Hofer, Pseudoholomorphic curves in symplectizations with applications to the Weinstein conjecture in dimension three, Inventiones Math. 114 (1993) 515563 MathReview

[17] R C Kirby, W B R Lickorish, Prime knots and concordance, Math. Proc. Cam. Phil. Soc. 86 (1979) 437-441 MathReview

[18] W B R Lickorish, Prime knots and tangles, Trans. Amer. Math. Soc. 267 (1981) 321-332 MathReview

[19] L Ng, Maximal Thurston-Bennequin number of two-bridge links, Algebr. Geom. Topol. 1 (2001) 427-434 MathReview

[20] L Ng, Computable Legendrian invariants, Topology 42 (2003) 55-82 MathReview

[21] L Ng, Framed knot contact homology arXiv:math.GT/0407071

[22] D Rolfsen, Knots and Links, Publish or Perish Press (1976) MathReview

[23] J Sabloff, Legendrian Knot Theory, book in preparation

[24] J Sabloff, Augmentations and rulings of Legendrian knots, International Math. Res. Notices 19 (2005) 1157-1180 MathReview

[25] J Sabloff, Duality for Legendrian contact homology, arXiv:math.SG/0508187

[26] N Yufa, Thurston-Bennequin invariant of Legendrian knots, senior thesis, MIT (2001) 